\title{
Qualitative Behaviour of Solutions in Two Models of Thin Liquid Films
}

\author{
Matthew Michal, ${ }^{1}$ Marina Chugunova, ${ }^{1}$ and Roman Taranets ${ }^{2}$ \\ ${ }^{1}$ Institute of Mathematical Sciences, Claremont Graduate University, 710 N. College Avenue, Claremont, CA 91711, USA \\ ${ }^{2}$ Department of Mathematics, University of California, Los Angeles, 520 Portola Plaza, Math Sciences Building 6363, \\ Los Angeles, CA 90095, USA
}

Correspondence should be addressed to Marina Chugunova; marina.chugunova@cgu.edu

Received 7 March 2016; Accepted 5 April 2016

Academic Editor: Jingxue Yin

Copyright (c) 2016 Matthew Michal et al. This is an open access article distributed under the Creative Commons Attribution License, which permits unrestricted use, distribution, and reproduction in any medium, provided the original work is properly cited.

For the thin-film model of a viscous flow which originates from lubrication approximation and has a full nonlinear curvature term, we prove existence of nonnegative weak solutions. Depending on initial data, we show algebraic or exponential dissipation of an energy functional which implies dissipation of the solution arc length that is a well known property for a Hele-Shaw flow. For the classical thin-film model with linearized curvature term, under some restrictions on parameter and gradient values, we also prove analytically the arc length dissipation property for positive solutions. We compare the numerical solutions for both models, with nonlinear and with linearized curvature terms. In regimes when solutions develop finite time singularities, we explain the difference in qualitative behaviour of solutions.

\section{Introduction}

Fluid flow where advective inertial forces are small compared with viscous forces can be described as a Stokes flow. A Stokes flow model is given by

$$
\begin{aligned}
\nabla p & =\mu \nabla^{2} \mathbf{u}+\mathbf{f}, \\
\nabla \cdot \mathbf{u} & =0,
\end{aligned}
$$

where $p$ is the pressure, $\mathbf{u}$ is the velocity of the fluid, $\mu$ is the dynamical viscosity, and $\mathbf{f}$ is an applied force.

In the special case when viscous fluid is moving slowly through a porous medium, one can simplify the Stokes model by introducing a Hele-Shaw regime. Consider a Hele-Shaw flow, where $x$ and $y$ directions are parallel to flat plates, and the $z$ direction is perpendicular to the plates. Also, there is only a small gap of $2 H$ between the plates that are at $z= \pm H$. Under these assumptions, the Hele-Show flow is guided by the system of equations:

$$
\begin{aligned}
\mathbf{u} & =\nabla p \frac{z^{2}-H^{2}}{2 \mu}, \\
\nabla^{2} p & =0, \\
\nabla p \cdot \vec{n} & =0,
\end{aligned}
$$

where the pressure $p=p(x, y, t)$, the velocity of the fluid $\mathbf{u}=\mathbf{u}(x, y, t)$, the dynamical viscosity $\mu$ has a constant value, and $\vec{n}$ is the normal vector to the fluid surface. The HeleShaw model describes viscous flow dynamics when a drop of liquid is pressed between two plates. This model is useful both for oil companies to better understand the process of secondary recovery of oil fields [1] and for environmental agencies attempting to analyze the flow of water moving through the soil [2].

It is well known [3] that solutions for the Hele-Shaw model, for some class of initial data, can develop singularities in finite time known as "fingering phenomenon." An interesting result was obtained by Constantin et al. [4]. It was shown that a thin neck of fluid between two larger droplets will not break in a finite time. Using the lubrication approximation approach, the authors proved that the minimum width of this neck decreases like $1 / t^{4}$ for large enough time $t$. Likewise, Almgren [5] showed that singularities can develop in flows driven only by surface tension in Hele-Shaw models.

In 2001, Hernández-Machado et al. developed a theory to predict the forced evolution of a liquid-air interface in a HeleShall cell [6]. Ruyer-Quil developed a Hele-Shaw model that 
corrects Darcy's law for inertial forces [7]. Later in 2002, Lee et al. described the pinch-off in mixtures of fluids and the linear stability of steady states [8]. He also studied the interface dynamics for certain types of fluid mixtures. At about the same time, Crowdy published a method for determining exact solutions in a rotating Hele-Shaw cell when the initial interface is a fluid annulus [9]. Also, at that time, two groups of mathematicians presented two different points of view on applicability of Hele-Shaw models to study instabilities in viscous flow dynamics. Böckmann and Müller showed the example where the slow flow could evolve properly in a two-dimensional Navier-Stokes model exhibiting previously experimentally observed fingering phenomenon but at the same time failed to obey Hele-Shaw model where no finite or infinite time singularity was formed [10]. Martin et al. found the solution for this disagreement in 2002 and showed that the instability actually can be described by a Hele-Shaw model if the equations include a chemical reaction term [11].

In lubrication approximation regime, namely, when the film thickness $H$ is much less than the length of the flow $L$ (i.e., the small parameter is introduced by $\epsilon=H / L \ll 1$ ), a coating flow can be modeled by a thin-film equation which, in the general-slip form, is given by

$$
h_{t}+\left(h^{n} h_{x x x}\right)_{x}=0 \text { (linearized curvature model), }
$$

where $h=h(x, t)$ is the time-dependent thickness of the thin fluid film and the value of the order of the nonlinearity $n$ depends on the slip conditions between the viscous liquid and the solid interface. With the order of the nonlinearity $n=1$ (which corresponds to the no-slip regime), this equation was derived for the lubrication limit of Hele-Shaw flow in [12]. The existence of nonnegative weak solutions, their qualitative behaviour, and regularity for (3) were rigorously studied in [13-15]. The asymptotic analysis results for classical and weak solutions of the thin-film equation were obtained in $[16,17]$. One of the most well known and still open questions is the uniqueness of strong nonnegative solutions for (3). Some results in this direction, for particular classes of initial data, can be found in $[18,19]$.

In this paper, firstly, we will analyze a general-slip model (3) with nonlinearity $n>0$. Secondly, instead of the linearized curvature term $\kappa \approx h_{x x}$ (which was used to obtain (3), the linearized curvature model), we will introduce the full nonlinear curvature expression $h_{t}+\left(h^{n}\left(\left(1+h_{x}^{2}\right)^{-1 / 2} h_{x}\right)_{x x}\right)_{x}=$ 0 (nonlinear curvature model). This intermediate asymptotic lubrication model allows us to use initial data with large values of the gradient. Recall that the classical thin-film model with linearized curvature term restricts initial data to small gradient values; that is, $\left|h_{x}\right| \ll 1$.

Our paper has the following structure. In Section 2, using a priori energy estimates, we will prove existence of nonnegative weak solutions for the nonlinear curvature model. In Section 3, we will obtain rate of energy dissipation along weak solutions constructed in Section 2. In Section 4, we will prove arc length dissipation property for some class of positive solutions for the linearized curvature model and, finally, in Section 5, we will compare numerical solutions for two models for the special choice of initial data and parameters which lead to formations of finite time singularities.

\section{Existence of Weak Solutions in Nonlinear Curvature Model}

We consider the thin-film equation with full nonlinear expression for the curvature term; namely,

$$
h_{t}+\left(|h|^{n}\left(f\left(h_{x}\right) h_{x}\right)_{x x}\right)_{x}=0 \quad \text { in } Q_{T}
$$

with initial data

$$
h(x, 0)=h_{0}(x)
$$

coupled with the boundary conditions

$$
h_{x}( \pm a, t)=\left(f\left(h_{x}\right) h_{x}\right)_{x x}( \pm a, t)=0 \text {, }
$$

where $f(z):=\left(1+z^{2}\right)^{-1 / 2}$ and $\Omega=(-a, a), Q_{T}=\Omega \times(0, T)$, $T>0$. Obviously, we have the mass conservation

$$
\int_{\Omega} h(x, t) d x=\int_{\Omega} h_{0}(x) d x=M .
$$

Formally, multiplying (4) by $-\left(f\left(h_{x}\right) h_{x}\right)_{x}$, after integrating over $\Omega$, we obtain

$$
\frac{d}{d t} \int_{\Omega} \frac{1}{f\left(h_{x}\right)} d x+\int_{\Omega}|h|^{n}\left(f\left(h_{x}\right) h_{x}\right)_{x x}^{2} d x=0,
$$

whence we find the arc length dissipation immediately

$$
\int_{\Omega} \sqrt{1+h_{x}^{2}} d x \leqslant \int_{\Omega} \sqrt{1+h_{0 x}^{2}} d x
$$

For numerical simulations of this arc length dissipation property (see Figure 1), obtained above, we use as initial data $h_{0}=\sin (x-\pi / 2) / 4+1 / 2$ and apply stable semi-implicit in time pseudospectral method developed in [20].

Definition 1. Let $n>0$. A generalized weak solution of problem (4)-(6) is a function $h$ satisfying

$$
\begin{aligned}
h & \in C\left(\bar{Q}_{T}\right) \cap L^{\infty}\left(0, T ; W_{1}^{1}(\Omega)\right), \\
h_{t} & \in L^{2}\left(0, T ;\left(H^{1}(\Omega)\right)^{\prime}\right), \\
|h|^{n / 2}\left(f\left(h_{x}\right) h_{x}\right)_{x x} & \in L^{2}\left(\mathscr{P}_{T}\right),
\end{aligned}
$$

where $\mathscr{P}_{T}:=\bar{Q}_{T} \backslash(\{h=0\} \cup\{t=0\})$ and $h$ satisfies (4) in the following sense:

$$
\begin{aligned}
& \int_{0}^{T}\left\langle h_{t}, \phi\right\rangle_{\left(H^{1}\right)^{\prime}, H^{1}} d t \\
& \quad-\iint_{\mathscr{P}_{T}}|h|^{n}\left(f\left(h_{x}\right) h_{x}\right)_{x x} \phi_{x} d x d t=0
\end{aligned}
$$

for all $\phi \in L^{2}\left(0, T ; H^{1}(\Omega)\right)$, and

$$
h(\cdot, t) \longrightarrow h_{0}(\cdot) \text { strongly in } L^{2}(\Omega) \quad \text { as } t \longrightarrow 0 .
$$



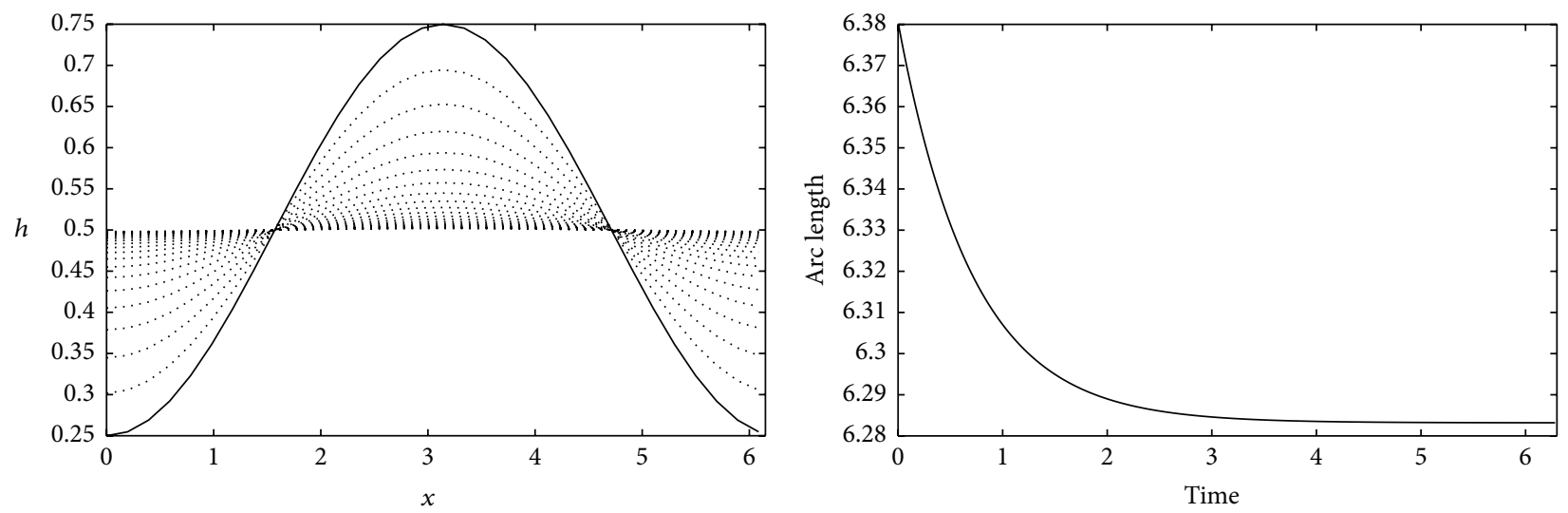

FIGURE 1: Arc length dissipation for a numerical solution when $n=1 / 3$.

Theorem 2 (existence of nonnegative weak solutions). Assume that $n>0$ and $h_{0} \in W_{1}^{1}(\Omega), h_{0} \geqslant 0, h_{0}^{\alpha-n+2} \in L^{1}(\Omega)$ for any $\alpha \in[-1 / 2,1)$. Then, for any time $T>0$, there exists a nonnegative generalized weak solution $h$ in the sense of Definition 1 such that

$$
\begin{aligned}
& h^{\alpha-n+2} \in L^{\infty}\left(0, T ; L^{1}(\Omega)\right) \quad \text { for any } \alpha \in\left[-\frac{1}{2}, 1\right), \\
& f^{3 / 2}\left(h_{x}\right)\left(h^{(\alpha+2) / 2}\right)_{x x} \in L^{2}\left(Q_{T}\right), \\
& f^{3 / 4}\left(h_{x}\right)\left(h^{(\alpha+2) / 4}\right)_{x} \in L^{4}\left(Q_{T}\right) \\
& \text { for any } \alpha \in\left(-\frac{1}{2}, 1\right), \\
& \sqrt{1+h_{x}^{2}(\cdot, t)} \longrightarrow \sqrt{1+h_{0 x}^{2}(\cdot)} \text { strongly in } L^{1}(\Omega)
\end{aligned}
$$$$
\text { as } t \longrightarrow 0 \text {. }
$$

Proof of Theorem 2. Given $\varepsilon>0, \delta>0$, and $\sigma>0$, an approximated parabolic problem that we consider is

$$
h_{t}+\left(g_{\varepsilon \delta}(h)\left(f_{\sigma}\left(h_{x}\right) h_{x}\right)_{x x}\right)_{x}=0 \text {, }
$$

with the initial data

$$
h(x, 0)=h_{0 \varepsilon \delta \sigma}(x)
$$

coupled with the boundary conditions

$$
h_{x}( \pm a, t)=\left(f\left(h_{x}\right) h_{x}\right)_{x x}( \pm a, t)=0 \text {, }
$$

where

$$
\begin{aligned}
g_{\varepsilon \delta}(z) & =\frac{|z|^{n+4}}{z^{4}+\delta|z|^{n}}+\varepsilon, \\
f_{\sigma}(z) & =f(z)+\sigma
\end{aligned}
$$

$$
\begin{aligned}
& 0<h_{0, \varepsilon \delta \sigma} \in C^{4+\alpha}(\bar{\Omega}): \\
& h_{0, \varepsilon \delta \sigma} \longrightarrow h_{0, \delta \sigma}>0 \text { strongly in } H^{1}(\Omega) \\
& \text { as } \varepsilon \longrightarrow 0 \text {, } \\
& h_{0, \delta \sigma} \longrightarrow h_{0, \sigma} \text { strongly in } H^{1}(\Omega) \quad \text { as } \delta \longrightarrow 0 \text {, } \\
& h_{0, \delta \sigma} \geqslant h_{0, \sigma}+\delta^{\theta}, \quad \theta \in\left(0, \frac{2}{5}\right) \text {, } \\
& h_{0, \sigma} \longrightarrow h_{0} \text { strongly in } W_{1}^{1}(\Omega) \text {, } \\
& \sigma\left\|h_{0 \sigma, x}\right\|_{2}^{2} \longrightarrow 0 \\
& \text { as } \sigma \longrightarrow 0 \text {. }
\end{aligned}
$$

Multiplying (14) by $-\left(f_{\sigma}\left(h_{x}\right) h_{x}\right)_{x}$, after integrating over $\Omega$, we obtain

$$
\begin{aligned}
& \frac{d}{d t} \int_{\Omega}\left(\frac{1}{f\left(h_{x}\right)}+\frac{\sigma}{2} h_{x}^{2}\right) d x \\
& \quad+\int_{\Omega} g_{\varepsilon \delta}(h)\left(f_{\sigma}\left(h_{x}\right) h_{x}\right)_{x x}^{2} d x=0 .
\end{aligned}
$$

Let $G_{\varepsilon \delta}(z)$ be such that $G_{\varepsilon \delta}^{\prime \prime}(z)=1 / g_{\varepsilon \delta}(z)>0$. Multiplying (14) by $G_{\varepsilon \delta}^{\prime}(h)$ (we have enough regularity because our solutions are classical), after integrating over $\Omega$, we obtain

$$
\begin{aligned}
& \frac{d}{d t} \int_{\Omega} G_{\varepsilon \delta}(h) d x+\sigma \int_{\Omega} h_{x x}^{2} d x+\int_{\Omega} f^{3}\left(h_{x}\right) h_{x x}^{2} d x \\
& \quad=0
\end{aligned}
$$

Using a priori estimates (18) and (19) and local in time existence of a classical solution for (14) (local existence can be shown by application of Galerkin method [21]), following [13], we take $\varepsilon \rightarrow 0$. As a result, we obtain a unique positive classical solution $h_{\delta \sigma}$ in $Q_{T}$ for any $T>0$; that is, $0<h_{\delta \sigma} \epsilon$ $C_{x, t}^{4,1}\left(Q_{T}\right)$. 
Let $\widetilde{G}_{\delta}(z)$ be such that $\widetilde{G}_{\delta}^{\prime \prime}(z)=z^{\alpha} / g_{\delta}(z)>0$ for all $z>0$. Multiplying (14) with $\varepsilon=0$ by $\widetilde{G}_{\delta}^{\prime}(h)$, after integrating over $\Omega$, we obtain

$$
\begin{aligned}
& \frac{d}{d t} \int_{\Omega} \widetilde{G}_{\delta}(h) d x \\
& \quad+\sigma \int_{\Omega}\left(h^{\alpha} h_{x x}^{2}-\alpha(\alpha-1) h^{\alpha-2} h_{x}^{4}\right) d x \\
& \quad+\int_{\Omega} h^{\alpha} f^{3}\left(h_{x}\right) h_{x x}^{2} d x \\
& \quad-\alpha(\alpha-1) \int_{\Omega} h^{\alpha-2} F\left(h_{x}\right) h_{x} d x=0,
\end{aligned}
$$

where

$$
\begin{aligned}
F(z) & :=\int_{0}^{z} s^{2} f^{3}(s) d s \\
& =\ln \left(z+\sqrt{1+z^{2}}\right)-\frac{z}{\sqrt{1+z^{2}}} .
\end{aligned}
$$

As

$$
\begin{aligned}
& \int_{\Omega} h^{\alpha-2} F\left(h_{x}\right) h_{x} d x=\frac{1}{1-\alpha} \\
& \cdot \int_{\Omega} h^{\alpha-1} h_{x}^{2} f^{3}\left(h_{x}\right) h_{x x} d x \\
& \int_{\Omega} h^{\alpha-1} h_{x}^{2} f^{3}\left(h_{x}\right) h_{x x} d x \leqslant\left(\int_{\Omega} h^{\alpha} f^{3}\left(h_{x}\right) h_{x x}^{2} d x\right)^{1 / 2} \\
& \cdot\left(\int_{\Omega} h^{\alpha-2} f^{3}\left(h_{x}\right) h_{x}^{4} d x\right)^{1 / 2},
\end{aligned}
$$

then

$$
\begin{aligned}
& \int_{\Omega} h^{\alpha-2} F\left(h_{x}\right) h_{x} d x \\
& \quad \leqslant \frac{1}{|1-\alpha|}\left(\int_{\Omega} h^{\alpha} f^{3}\left(h_{x}\right) h_{x x}^{2} d x\right)^{1 / 2} \\
& \quad \cdot\left(\int_{\Omega} h^{\alpha-2} f^{3}\left(h_{x}\right) h_{x}^{4} d x\right)^{1 / 2} .
\end{aligned}
$$

Using the inequality

$$
z^{4} f^{3}(z) \leqslant 3 z F(z) \quad \forall z \in \mathbb{R}^{1},
$$

we have

$$
\int_{\Omega} h^{\alpha-2} F\left(h_{x}\right) h_{x} d x \leqslant \frac{3}{(1-\alpha)^{2}} \int_{\Omega} h^{\alpha} f^{3}\left(h_{x}\right) h_{x x}^{2} d x .
$$

As a result, we obtain

$$
\begin{array}{r}
\frac{d}{d t} \int_{\Omega} \widetilde{G}_{\delta}(h) d x+\sigma \frac{1+2 \alpha}{1-\alpha} \int_{\Omega} h^{\alpha} h_{x x}^{2} d x \\
+\frac{1+2 \alpha}{1-\alpha} \int_{\Omega} h^{\alpha} f^{3}\left(h_{x}\right) h_{x x}^{2} d x \leqslant 0,
\end{array}
$$

where $\alpha \in[-1 / 2,1)$. Using a priori estimate (26), following $[22,23]$, we take $\delta \rightarrow 0$. As a result, we obtain a nonnegative strong solution $h_{\sigma}$ in $Q_{T}$ for any $T>0$; that is, $0 \leqslant h_{\sigma} \in C^{1}(\bar{\Omega})$ for a. e. $t>0$.

From (18)-(26), we obtain that

$$
\left\{\sqrt{1+h_{x}^{2}}\right\}_{\sigma>0}
$$

is uniformly bounded in $L^{\infty}\left(0, T ; L^{1}(\Omega)\right)$;

$$
\left\{h^{\alpha-n+2}\right\}_{\sigma>0}
$$

is uniformly bounded in $L^{\infty}\left(0, T ; L^{1}(\Omega)\right)$,

$$
\alpha \in\left[-\frac{1}{2}, 1\right) \text {; }
$$

$$
\left\{f^{3 / 2}\left(h_{x}\right)\left(h^{(\alpha+2) / 2}\right)_{x x}\right\}_{\sigma>0}
$$

is uniformly bounded in $L^{2}\left(Q_{T}\right), \quad \alpha \in\left(-\frac{1}{2}, 1\right)$;

$$
\left\{f^{3 / 4}\left(h_{x}\right)\left(h^{(\alpha+2) / 4}\right)_{x}\right\}_{\sigma>0}
$$

$$
\text { is uniformly bounded in } L^{4}\left(Q_{T}\right), \quad \alpha \in\left(-\frac{1}{2}, 1\right) \text {. }
$$

In particular, from (27), it follows that

$\{h\}_{\sigma>0}$ is uniformly bounded in $L^{\infty}\left(0, T ; W_{1}^{1}(\Omega)\right)$;

$$
\{h\}_{\sigma>0} \text { is uniformly bounded in } C\left(\bar{Q}_{T}\right) .
$$

Following [21], we let $\sigma \rightarrow 0$. As a result, we obtain the existence of a nonnegative weak solution $h$ in $Q_{T}$ for any $T>0$; that is, $0 \leqslant h \in C\left(\bar{Q}_{T}\right)$.

\section{Dissipation Rate of the Energy Functional}

Theorem 3 (asymptotic decay). Assume that $h$ is a nonnegative generalized weak solution from Theorem 2 . Then, there exists a constant $C_{0}>0$ depending only on initial data $h_{0}$ and $|\Omega|$ such that the solution $h$ satisfies

$$
\begin{aligned}
\int_{\Omega}\left(\sqrt{1+h_{x}^{2}}-1\right) d x \leqslant C_{0}(1+t)^{-1}, & \\
h(x, t) & \longrightarrow \frac{M}{|\Omega|} \quad \text { in } W_{1}^{1}(\Omega) \\
\text { as } t & \longrightarrow+\infty .
\end{aligned}
$$

Moreover, if $M>|\Omega|^{2}$, then there exists a constant $C_{1}>0$ depending only on initial data $h_{0}$ and $|\Omega|$, such that

$$
\begin{aligned}
& \int_{\Omega}\left(\sqrt{1+h_{x}^{2}}-1\right) d x \\
& \quad \leqslant \exp \left(-C_{1} t\right) \int_{\Omega}\left(\sqrt{1+h_{0 x}^{2}}-1\right) d x .
\end{aligned}
$$

Remark 4. In fact, Theorem 3 implies the existence of $T^{*}=$ $T^{*}\left(h_{0}\right) \geqslant 0$ such that $h(x, t)>0$ for all $t>T^{*}$. 
Remark 5. Using Galerkin method we can observe numerically that the arc length has exponential decay rate.

The exponential dissipation rate is illustrated in Figure 2 for initial data for $h_{0}=\sin (x-\pi / 2) / 4+1 / 2$ and the nonlinearity $n=1 / 3$.

Proof of Theorem 3. We consider a unique positive classical solution $h=h_{\delta \sigma}$. Let us denote

$$
\begin{aligned}
& L_{\sigma}[z]:=\sqrt{1+z^{2}}+\frac{\sigma}{2} z^{2}, \\
& L_{\sigma}^{\prime}[z]=z f_{\sigma}(z), \\
& L_{\sigma}^{\prime \prime}[z]=f^{3}(z)+\sigma .
\end{aligned}
$$

Next, we have

$$
\begin{aligned}
L_{\sigma}\left[h_{x}\right]= & L_{\sigma}\left[h_{x}\left(x_{0}\right)\right]+\int_{x_{0}}^{x}\left(L_{\sigma}\left[h_{x}\right]\right)_{x} d x \\
= & L_{\sigma}\left[h_{x}\left(x_{0}\right)\right]+\int_{x_{0}}^{x} L_{\sigma}^{\prime}\left[h_{x}\right] h_{x x} d x \\
= & L_{\sigma}\left[h_{x}\left(x_{0}\right)\right]+\int_{x_{0}}^{x} f_{\sigma}\left(h_{x}\right) h_{x} h_{x x} d x \\
= & L_{\sigma}\left[h_{x}\left(x_{0}\right)\right]+\left.f_{\sigma}\left(h_{x}\right) h_{x}^{2}\right|_{x_{0}} ^{x} \\
& -\left.\left(f_{\sigma}\left(h_{x}\right) h_{x}\right)_{x} h\right|_{x_{0}} ^{x} \\
& +\int_{x_{0}}^{x}\left(f_{\sigma}\left(h_{x}\right) h_{x}\right)_{x} h_{x} d x \\
= & f_{\sigma}\left(h_{x}\right) h_{x}^{2}-\left(f_{\sigma}\left(h_{x}\right) h_{x}\right)_{x} h \\
& +\int_{x_{0}}^{x}\left(f_{\sigma}\left(h_{x}\right) h_{x}\right)_{x} h_{x} d x-F\left(x_{0}\right) \\
= & 2 f_{\sigma}\left(h_{x}\right) h_{x}^{2}-\left(f_{\sigma}\left(h_{x}\right) h_{x} h\right)_{x} \\
& +\int_{x_{0}}^{x}\left(f_{\sigma}\left(h_{x}\right) h_{x}\right)_{x} h_{x} d x-F\left(x_{0}\right),
\end{aligned}
$$

where

$$
F(x):=-L_{\sigma}\left[h_{x}\right]+f_{\sigma}\left(h_{x}\right) h_{x}^{2}-\left(f_{\sigma}\left(h_{x}\right) h_{x}\right)_{x} h .
$$

Integrating this equality on $\Omega$, we deduce that

$$
\begin{gathered}
-\int_{\Omega}\left(L_{\sigma}\left[h_{x}\right]+F\left(x_{0}\right)\right) d x+2 \int_{\Omega} f_{\sigma}\left(h_{x}\right) h_{x}^{2} d x \\
=-\int_{\Omega} \int_{x_{0}}^{y}\left(f_{\sigma}\left(h_{x}\right) h_{x}\right)_{x x} h d x d y .
\end{gathered}
$$

We select a point $x_{0} \in \bar{\Omega}$ such that $h_{x}\left(x_{0}\right)=0$ and $h_{x x}\left(x_{0}\right) \geqslant$ 0 . Really, we know that we have at least two such points such that $h_{x}\left(x_{0}\right)=0$. Of course, if either $h_{x x}(-a)$ or $h_{x x}(a)$ is nonnegative, then we are done by choosing $x_{0}$ to be that endpoint. If, however, both $h_{x x}(-a)$ and $h_{x x}(a)$ are negative,

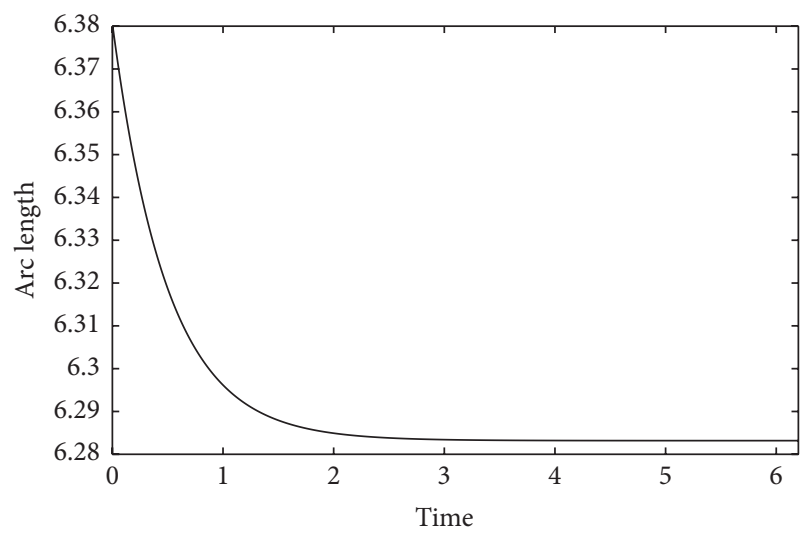

Figure 2: Exponential decay of the arc length for the numerical solution with $C_{1}=2.017$.

then there exists at least one $x_{0} \in \Omega$ for which $h_{x}\left(x_{0}\right)=0$ and $h_{x x}\left(x_{0}\right) \geqslant 0$. As a result, $F\left(x_{0}\right) \leqslant-1$. Then, we have

$$
\begin{gathered}
\int_{\Omega}\left(1+\frac{1}{f\left(h_{x}\right)}\left(1-2 f^{2}\left(h_{x}\right)\right)+\frac{3 \sigma}{2} h_{x}^{2}\right) d x \\
\leqslant-\int_{\Omega} \int_{x_{0}}^{y}\left(f_{\sigma}\left(h_{x}\right) h_{x}\right)_{x x} h d x d y .
\end{gathered}
$$

In view of the inequality $1+(1 / f(z))\left(1-2 f^{2}(z)\right) \geqslant 1 / f(z)-$ $1 \forall z \in \mathbb{R}^{1}$, we obtain that

$$
\begin{aligned}
& \int_{\Omega}\left(L_{\sigma}\left[h_{x}\right]-1\right) d x \\
& \quad \leqslant-\int_{\Omega} \int_{x_{0}}^{y}\left(f_{\sigma}\left(h_{x}\right) h_{x}\right)_{x x} h d x d y .
\end{aligned}
$$

Using the estimate

$$
\begin{aligned}
& -\int_{x_{0}}^{x}\left(f_{\sigma}\left(h_{x}\right) h_{x}\right)_{x x} h d x \\
& \quad \leqslant\left(\int_{\Omega} g_{\delta}(h)\left(f_{\sigma}\left(h_{x}\right) h_{x}\right)_{x x}^{2} d x\right)^{1 / 2} \\
& \quad \cdot\left(\int_{\Omega} \frac{h^{2}}{g_{\delta}(h)} d x\right)^{1 / 2}
\end{aligned}
$$

and taking into account $\int_{\Omega}\left(h^{2} / g_{\delta}(h)\right) d x \leqslant C_{0}=C_{0}\left(h_{0}\right)$ due to (19) with $\varepsilon=0$, we find that

$$
\begin{aligned}
& \left(\int_{\Omega}\left(L_{\sigma}\left[h_{x}\right]-1\right) d x\right)^{2} \\
& \quad \leqslant C_{0} \int_{\Omega} g_{\delta}(h)\left(f_{\sigma}\left(h_{x}\right) h_{x}\right)_{x x}^{2} d x .
\end{aligned}
$$

Thus, from (18), we arrive at

$$
\begin{aligned}
v_{\sigma}(t)+C_{0}^{-1} \int_{0}^{t} v_{\sigma}^{2}(s) d s \leqslant v_{\sigma}(0), \\
\text { where } v_{\sigma}(t):=\int_{\Omega}\left(L_{\sigma}\left[h_{x}\right]-1\right) d x .
\end{aligned}
$$


Letting $\sigma \rightarrow 0$, from this inequality, we obtain

$$
0 \leqslant v_{0}(t) \leqslant \frac{v_{0}(0)}{1+C_{0}^{-1} v_{0}(0) t} \longrightarrow 0 \text { as } t \longrightarrow+\infty
$$

and hence

$$
\int_{\Omega}\left(\sqrt{1+h_{x}^{2}}-1\right) d x \longrightarrow 0 \text { as } t \longrightarrow+\infty .
$$

Thus,

$$
h(x, t) \longrightarrow \frac{M}{|\Omega|} \text { in } W_{1}^{1}(\Omega) \quad \text { as } t \longrightarrow+\infty .
$$

Now, we show that

$$
\begin{array}{r}
\text { if } M>|\Omega|^{2} \text { then } 0 \leqslant v_{0}(t) \leqslant v_{0}(0) \exp \left\{-C_{1} t\right\} \\
\text { as } t \longrightarrow 0
\end{array}
$$

where $C_{1}$ is some positive constant.

Next, let $\mu>0$ to be fixed later. Note that (41) implies

$$
\int_{\Omega} g_{\delta}(h)\left(f_{\sigma}\left(h_{x}\right) h_{x}\right)_{x x}^{2} d x \geqslant \mu C_{0}^{-1} v_{\sigma}(t)
$$

$$
\text { if } v_{\sigma}(t) \geqslant \mu \text {. }
$$

If $v_{\sigma}(t)<\mu$, then

$$
\left|h-\frac{M}{|\Omega|}\right| \leqslant \int_{\Omega}\left|h_{x}\right| d x \leqslant \int_{\Omega} \sqrt{1+h_{x}^{2}} d x<\mu+|\Omega|,
$$

whence

$$
\inf \{h\} \geqslant h_{1}:=\frac{M}{|\Omega|}-\mu-|\Omega|>0 \quad \text { if } \mu<\frac{M-|\Omega|^{2}}{|\Omega|} .
$$

Thus, if $0 \leqslant v_{\sigma}(t)<\mu<\left(M-|\Omega|^{2}\right) /|\Omega|$, then

$$
\begin{gathered}
\int_{\Omega} g_{\delta}(h)\left(f_{\sigma}\left(h_{x}\right) h_{\mathrm{x}}\right)_{x x}^{2} d x \\
\geqslant g_{\delta}\left(h_{1}\right) \int_{\Omega}\left(f_{\sigma}\left(h_{x}\right) h_{x}\right)_{x x}^{2} d x \\
\geqslant g_{\delta}\left(h_{1}\right)\left(\frac{\pi}{|\Omega|}\right)^{4} v_{\sigma}(t) .
\end{gathered}
$$

Here, we used the fact that $g_{\delta}(z)$ is increasing on $[0, \infty)$ for any $\delta>0$ and the inequalities

$$
\begin{aligned}
v_{\sigma}(t) & \leqslant \int_{\Omega} \frac{1}{f\left(h_{x}\right)}\left(f_{\sigma}\left(h_{x}\right) h_{x}\right)^{2} d x \\
& \leqslant|\Omega|\left(\int_{\Omega} \frac{1}{f\left(h_{x}\right)} d x\right)\left(\int_{\Omega}\left(f_{\sigma}\left(h_{x}\right) h_{x}\right)_{x}^{2} d x\right) \\
& \leqslant \frac{|\Omega|^{3}}{\pi^{2}}\left(\int_{\Omega} \frac{1}{f\left(h_{x}\right)} d x\right)\left(\int_{\Omega}\left(f_{\sigma}\left(h_{x}\right) h_{x}\right)_{x x}^{2} d x\right) \\
& \leqslant \frac{|\Omega|^{3}(\mu+|\Omega|)}{\pi^{2}} \int_{\Omega}\left(f_{\sigma}\left(h_{x}\right) h_{x}\right)_{x x}^{2} d x ;
\end{aligned}
$$

that is,

$$
\begin{aligned}
\int_{\Omega}\left(f_{\sigma}\left(h_{x}\right) h_{x}\right)_{x x}^{2} d x & \geqslant\left(\frac{\pi}{|\Omega|}\right)^{4} \int_{\Omega}\left(f_{\sigma}\left(h_{x}\right) h_{x}\right)^{2} d x \\
& \geqslant \frac{\pi^{2}}{|\Omega|^{3}(\mu+|\Omega|)} v_{\sigma}(t) .
\end{aligned}
$$

As a result, from (47) and (50), we find that

$$
\int_{\Omega} g_{\delta}(h)\left(f_{\sigma}\left(h_{x}\right) h_{x}\right)_{x x}^{2} d x \geqslant C_{1} v_{\sigma}(t)
$$

where

$$
C_{1}=\sup _{\mu} \min \left\{\mu C_{0}^{-1}, g_{\delta}\left(h_{1}\right) \frac{\pi^{2}}{|\Omega|^{3}(\mu+|\Omega|)}\right\} .
$$

Thus, from (18), we arrive at

$$
v_{\sigma}(t)+C_{1} \int_{0}^{t} v_{\sigma}(s) d s \leqslant v_{\sigma}(0) .
$$

Letting $\delta \rightarrow 0$ and $\sigma \rightarrow 0$, from this inequality, we obtain (46).

\section{Arc length Dissipation of Positive Solutions in Linearized Curvature Model}

The time evolution behaviour of the arc length for a positive solution of the thin-film equation is bounded from above; namely, $\int_{\Omega}\left(\sqrt{1+h_{x}^{2}}-1\right) d x \leq 1 / 2 \int_{\Omega} h_{x}^{2} d x$, which dissipates to zero as time goes to infinity. Our goal is to show that the arc length also dissipates in time. Consider the equation

$$
h_{t}+\left(h^{n} h_{x x x}\right)_{x}=0 \quad \text { for }(x, t) \in Q_{T}
$$

coupled with the initial condition

$$
h(x, 0)=h_{0}(x)
$$

and the boundary conditions

$$
h_{x}=h_{x x x}=0 \quad \text { on } \partial \Omega .
$$

For future calculations,

$$
\begin{aligned}
0 \leq f^{n}(z) \leq 1 & \text { for any } n>0, \forall z \in \mathbb{R}^{1}, \\
1-z^{2} f^{2}(z) & =f^{2}(z) \geq 0 \Longrightarrow 0 \leq z^{2} f^{2}(z) \leq 1, \\
\left(f\left(h_{x}\right) h_{x}\right)_{x} & =f^{3}\left(h_{x}\right) h_{x x}, \\
\left(f^{3}\left(h_{x}\right) h_{x x}\right)_{x} & =-3 f^{5}\left(h_{x}\right) h_{x} h_{x x}^{2}+f^{3}\left(h_{x}\right) h_{x x x} .
\end{aligned}
$$

Theorem 6. Assume that $h_{0} \in C^{4+\alpha}(\bar{\Omega}), h_{0}>0$, and

$$
0<n<n^{*}:=\frac{3}{4} \text {. }
$$


Then, there exists a classical solution $h \in C_{x, t}^{4,1}\left(\bar{Q}_{T}\right)$ and a finite $\delta=\delta(n)>0$ such that the arc length dissipates; namely,

$$
\begin{aligned}
& \int_{\Omega} \sqrt{1+h_{x}^{2}} d x+C(\delta) \iint_{Q_{T}} f^{3}\left(h_{x}\right) h^{n} h_{x x x}^{2} d x d t \\
& \quad \leq \int_{\Omega} \sqrt{1+h_{0 x}^{2}} d x
\end{aligned}
$$

provided

$$
\left|h_{x}\right| \leq \delta
$$

Existence of classical solutions which satisfy condition (62) follows from parabolic Schauder estimates for their derivatives [24].

Proof of Theorem 6. If $h_{0}>0$, then there is a classical solution that exists locally in time [25]. If we multiply (56) by $\left(f\left(h_{x}\right) h_{x}\right)_{x}$ and integrate on $\Omega$, then we arrive at an equation describing the change in the arc length of the fluid:

$$
\begin{aligned}
& \int_{\Omega} h_{t}\left(f\left(h_{x}\right) h_{x}\right)_{x} d x+\int_{\Omega}\left(h^{n} h_{x x x}\right)_{x} f^{3}\left(h_{x}\right) h_{x x} d x \\
& =0 \\
& -\int_{\Omega} f\left(h_{x}\right) h_{x} h_{x t} d x-\int_{\Omega}\left(f^{3}\left(h_{x}\right) h_{x x}\right)_{x} h^{n} h_{x x x} d x \\
& =0 \\
& -\int_{\Omega} \frac{h_{x} h_{x t}}{\sqrt{1+h_{x}^{2}}} d x \\
& \quad-\int_{\Omega}\left(-3 f^{5}\left(h_{x}\right) h_{x} h_{x x}^{2}+f^{3}\left(h_{x}\right) h_{x x x}\right) h^{n} h_{x x x} d x \\
& \quad=0, \\
& \int_{\Omega} 3 f^{5}\left(h_{x}\right) h^{n} h_{x} h_{x x}^{2} h_{x x x} d x-\int_{\Omega} f^{3}\left(h_{x}\right) h^{n} h_{x x x}^{2} d x \\
& \quad=\frac{d}{d t} \int_{\Omega} \sqrt{1+h_{x}^{2}} d x .
\end{aligned}
$$

Therefore, for classical solutions to be dissipative, the second integral must be greater than the first one.

We rewrite this equality in a more convenient form:

$$
\begin{aligned}
& \frac{d}{d t} \int_{\Omega} \frac{1}{f\left(h_{x}\right)} d x+\int_{\Omega} h^{n} f^{-3}\left(h_{x}\right)\left(f\left(h_{x}\right) h_{x}\right)_{x x}^{2} d x \\
& \quad=3 \int_{\Omega} h^{n} f^{-4}\left(h_{x}\right) h_{x}\left(f\left(h_{x}\right) h_{x}\right)_{x}^{2}\left(f\left(h_{x}\right) h_{x}\right)_{x x} d x .
\end{aligned}
$$

Using the integration by parts, we find that

$$
\begin{gathered}
\frac{d}{d t} \int_{\Omega} \frac{1}{f\left(h_{x}\right)} d x+\int_{\Omega} h^{n} f^{-3}\left(h_{x}\right)\left(f\left(h_{x}\right) h_{x}\right)_{x x}^{2} d x \\
\quad+\int_{\Omega} h^{n} f^{-5}\left(h_{x}\right)\left(1+5 h_{x}^{2}\right)\left(f\left(h_{x}\right) h_{x}\right)_{x}^{4} d x \\
=-n \int_{\Omega} h^{n-1} f^{-4}\left(h_{x}\right) h_{x}^{2}\left(f\left(h_{x}\right) h_{x}\right)_{x}^{3} d x .
\end{gathered}
$$

Before we continue, notice that

$$
\begin{aligned}
& 3 \int_{\Omega} f^{5}\left(h_{x}\right) h^{n} h_{x} h_{x x}^{2} h_{x x x} d x \\
& \leq 3\left(\int_{\Omega} f^{3}\left(h_{x}\right) h^{n} h_{x x x}^{2} d x\right)^{1 / 2} \\
& \cdot\left(\int_{\Omega} f^{7}\left(h_{x}\right) h^{n} h_{x}^{2} h_{x x}^{4} d x\right)^{1 / 2} .
\end{aligned}
$$

Next, we show that

$$
\begin{aligned}
& \int_{\Omega} f^{7}\left(h_{x}\right) h^{n} h_{x}^{2} h_{x x}^{4} d x \\
& \quad \leq C_{0}(\delta) \int_{\Omega} f^{3}\left(h_{x}\right) h^{n} h_{x x x}^{2} d x .
\end{aligned}
$$

Let us denote

$$
\begin{aligned}
G(z) & :=\frac{1}{3} z^{3} f^{3}(z)-\frac{1}{5} z^{5} f^{5}(z) \\
& =\frac{1}{5} z^{3} f^{3}(z)\left(\frac{2}{3}+f^{2}(z)\right) \Longrightarrow G^{\prime}(z) \\
& =z^{2} f^{7}(z) .
\end{aligned}
$$

Then,

$$
\begin{aligned}
\int_{\Omega} G^{\prime}\left(h_{x}\right) h^{n} h_{x x}^{4} d x= & -n \int_{\Omega} G\left(h_{x}\right) h_{x} h^{n-1} h_{x x}^{3} d x \\
& -3 \int_{\Omega} G\left(h_{x}\right) h^{n} h_{x x}^{2} h_{x x x} d x .
\end{aligned}
$$

By Hölder inequality, we find that

$$
\begin{gathered}
\int_{\Omega} G\left(h_{x}\right) h_{x} h^{n-1} h_{x x}^{3} d x \leq\left(\int_{\Omega} G^{\prime}\left(h_{x}\right) h^{n} h_{x x}^{4} d x\right)^{3 / 4} \\
\cdot\left(\int_{\Omega} \frac{\left(G\left(h_{x}\right)\right)^{4}}{\left(G^{\prime}\left(h_{x}\right)\right)^{3}} h^{n-4} h_{x}^{4} d x\right)^{1 / 4}, \\
\int_{\Omega} G\left(h_{x}\right) h^{n} h_{x x}^{2} h_{x x x} d x \\
\leq\left(\int_{\Omega} f^{3}\left(h_{x}\right) h^{n} h_{x x x}^{2} d x\right)^{1 / 2} \\
\cdot\left(\int_{\Omega} \frac{\left(G\left(h_{x}\right)\right)^{2}}{f^{3}\left(h_{x}\right)} h^{n} h_{x x}^{4} d x\right)^{1 / 2}
\end{gathered}
$$

where

$$
\begin{aligned}
\frac{(G(z))^{4}}{\left(G^{\prime}(z)\right)^{3}} & =\frac{z^{6}}{625 f^{9}(z)}\left(\frac{2}{3}+f^{2}(z)\right)^{4} \\
\frac{(G(z))^{2}}{f^{3}(z)} & =\frac{z^{6} f^{3}(z)}{25}\left(\frac{2}{3}+f^{2}(z)\right)^{2}
\end{aligned}
$$



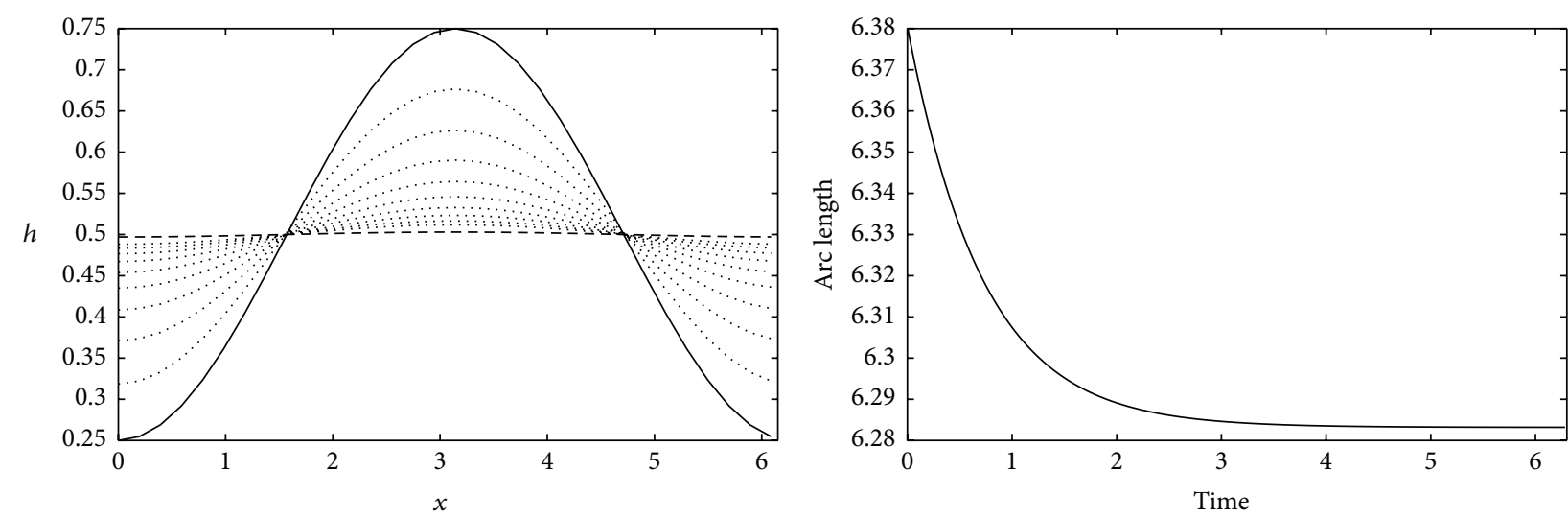

FIgURE 3: Arc length dissipation for the numerical solution with $n=1 / 2$ and $N=32$.

In particular, due to $\left|h_{x}\right| \leq \delta$, we get

$$
\frac{(G(z))^{2}}{f^{3}(z)} \leq a_{1} G^{\prime}(z) \quad \text { for } a_{1} \geq\left(\frac{\delta^{2}\left(2 \delta^{2}+5\right)}{15}\right)^{2} .
$$

Let us denote

$$
F(z):=\frac{(G(z))^{4}}{\left(G^{\prime}(z)\right)^{3}}=\frac{z^{6}}{625 f^{9}(z)}\left(\frac{2}{3}+f^{2}(z)\right)^{4}
$$

Then,

$$
\begin{aligned}
\int_{\Omega} F\left(h_{x}\right) h^{n-4} h_{x}^{4} d x \\
=\frac{1}{3-n} \int_{\Omega} F^{\prime}\left(h_{x}\right) h^{n-3} h_{x}^{3} h_{x x} d x \\
\quad+\frac{3}{3-n} \int_{\Omega} F\left(h_{x}\right) h^{n-3} h_{x}^{2} h_{x x} d x .
\end{aligned}
$$

By Hölder inequality, we deduce that

$$
\begin{gathered}
\int_{\Omega} F^{\prime}\left(h_{x}\right) h^{n-3} h_{x}^{3} h_{x x} d x \leq\left(\int_{\Omega} G^{\prime}\left(h_{x}\right) h^{n} h_{x x}^{4} d x\right)^{1 / 4} \\
\cdot\left(\int_{\Omega} \frac{\left(F^{\prime}\left(h_{x}\right)\right)^{4 / 3}}{\left(G^{\prime}\left(h_{x}\right)\right)^{1 / 3}} h^{n-4} h_{x}^{4} d x\right)^{3 / 4}, \\
\int_{\Omega} F\left(h_{x}\right) h^{n-3} h_{x}^{2} h_{x x} d x \leq\left(\int_{\Omega} G^{\prime}\left(h_{x}\right) h^{n} h_{x x}^{4} d x\right)^{1 / 4} \\
\cdot\left(\int_{\Omega} \frac{\left(F\left(h_{x}\right)\right)^{4 / 3}}{\left(G^{\prime}\left(h_{x}\right)\right)^{1 / 3}\left|h_{x}\right|^{4 / 3}} h^{n-4} h_{x}^{4} d x\right)^{3 / 4} \cdot
\end{gathered}
$$

In particular, due to $\left|h_{x}\right| \leq \delta$, we get

$$
\frac{(F(z))^{4 / 3}}{\left(G^{\prime}(z)\right)^{1 / 3} z^{4 / 3}} \leq a_{2} F(z)
$$

$$
\text { for } a_{2} \geq\left(\frac{2 \delta^{4}+7 \delta^{2}+5}{15}\right)^{4 / 3} \text {, }
$$

$$
\frac{\left(F^{\prime}(z)\right)^{4 / 3}}{\left(G^{\prime}(z)\right)^{1 / 3}} \leq a_{3} F(z)
$$

$$
\text { for } a_{3} \geq\left(\frac{10 \delta^{4}+21 \delta^{2}+10}{5}\right)^{4 / 3} \text {. }
$$

Thus,

$$
\begin{aligned}
& \int_{\Omega} F\left(h_{x}\right) h^{n-4} h_{x}^{4} d x \\
& \quad \leq\left(\frac{a_{3}^{3 / 4}+3 a_{2}^{3 / 4}}{|3-n|}\right)^{4} \int_{\Omega} G^{\prime}\left(h_{x}\right) h^{n} h_{x x}^{4} d x .
\end{aligned}
$$

From this, we deduce that

$$
\int_{\Omega} G^{\prime}\left(h_{x}\right) h^{n} h_{x x}^{4} d x \leq a_{4} \int_{\Omega} f^{3}\left(h_{x}\right) h^{n} h_{x x x}^{2} d x
$$

where

$$
a_{4}=\frac{9(3-n)^{2} a_{1}}{\left(|3-n|-n\left(a_{3}^{3 / 4}+3 a_{2}^{3 / 4}\right)\right)^{2}}
$$

provided

$$
\begin{aligned}
\left|h_{x}\right| & <\delta_{1}:=\sqrt{\frac{1}{6(-7+\sqrt{-11+45 / n})}}, \\
0 & <n<n^{*}=\frac{3}{4} .
\end{aligned}
$$

As a result, we obtain that

$$
\frac{d}{d t} \int_{\Omega} \sqrt{1+h_{x}^{2}} d x+a_{5} \int_{\Omega} f^{3}\left(h_{x}\right) h^{n} h_{x x x}^{2} d x \leq 0,
$$



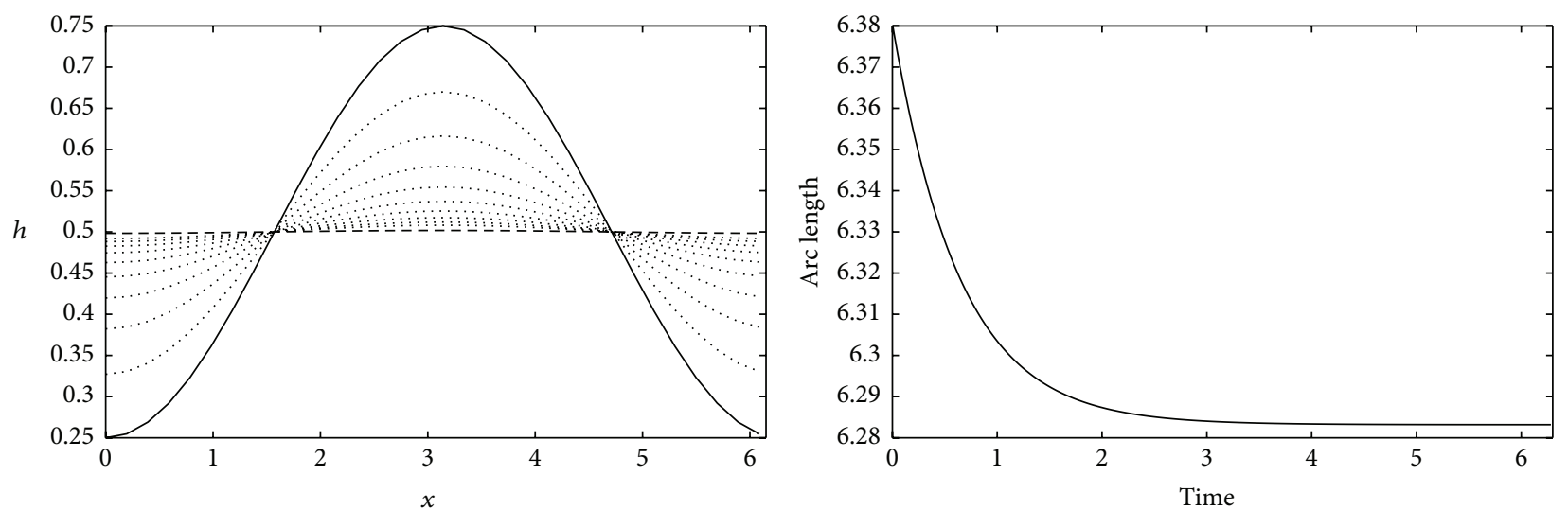

FIgURE 4: Arc length dissipation for the numerical solution with $n=1 / 3$ and $N=32$.
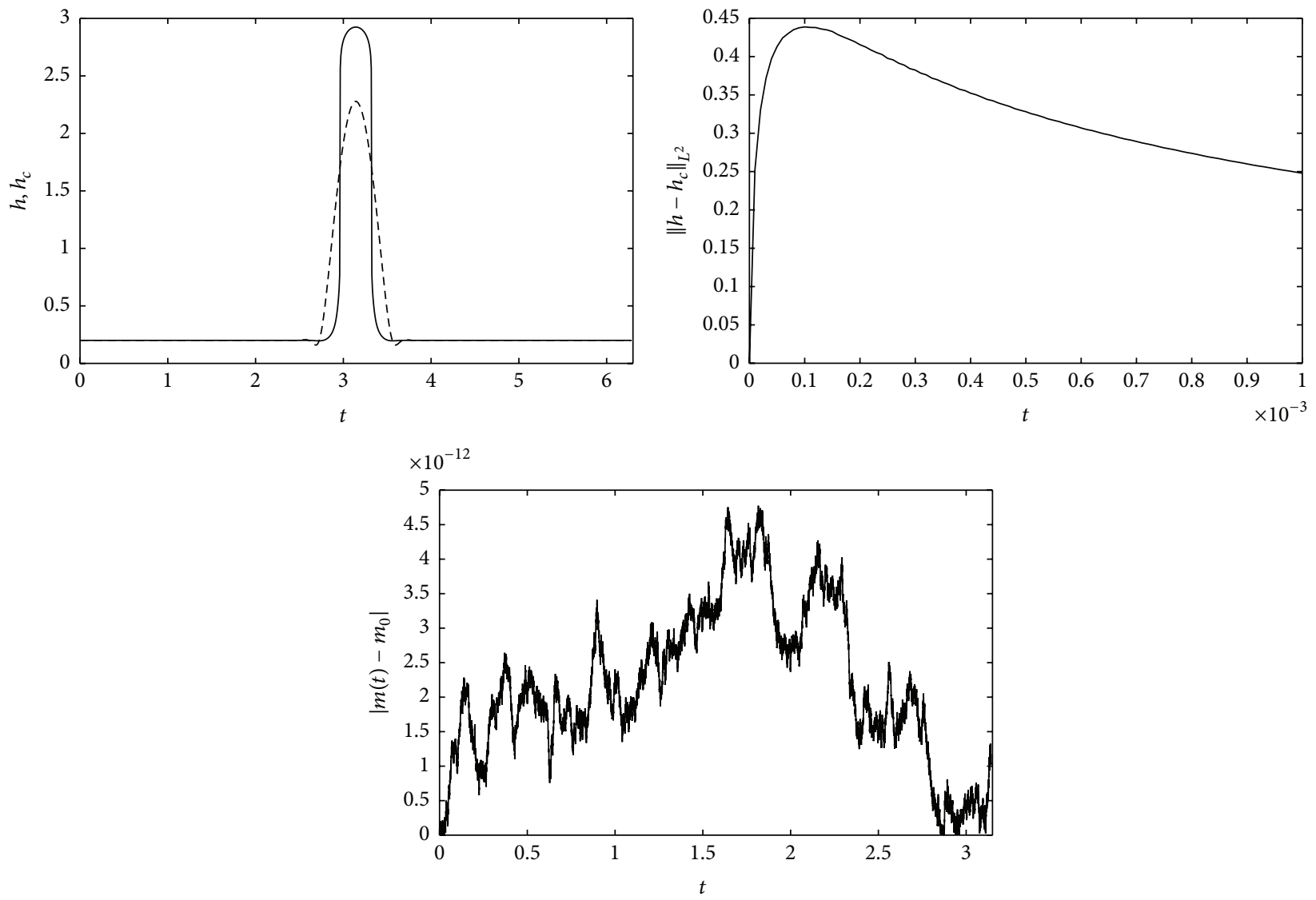

Figure 5: Comparison of solutions to (4) as the solid line and (56) as the dashed line for $n=2$ and $N=2^{8}$ when the solutions are at the maximum difference in their $L^{2}$ norms. Both numerical solutions are mass preserving.

where $a_{5}=1-3 a_{4}^{1 / 2} \geq 0$ and $a_{1}<(1 / 81)\left(\Rightarrow \delta<\delta_{2}:=\right.$ $(1 / 2) \sqrt{-5+\sqrt{115 / 3}} \approx 0.5458)$ provided

$$
\left|h_{x}\right|<\delta=\min \left\{\delta_{1}, \delta_{2}\right\}, \quad 0<n<n^{*} .
$$

We know from [13] that, for $n \geq 4$ and $h_{0}>0$, the solution for thin-film equation has the property $h(\cdot, t) \rightarrow$ $(1 /|\Omega|) \int h_{0}(x) d x$ uniformly as $t \rightarrow \infty$; this is why we believe that one can use the approximation $h^{n+4} /\left(h^{4}+\epsilon h^{n}\right)$ for $h^{n}$ for $\epsilon \ll 1$ to generalize our arc length dissipation result for strong nonnegative solutions.

To illustrate analytic results of arc length dissipation (see Figures 3 and 4) for the linearized curvature model numerically, we considered the problem on the domain $Q_{T}=[0,2 \pi] \times[0, \pi / 2]$. We discretized $\Omega$ using a uniform distribution with $2^{N}$ grid points for $N=10$ and applied Fourier spectral differentiation matrices with 

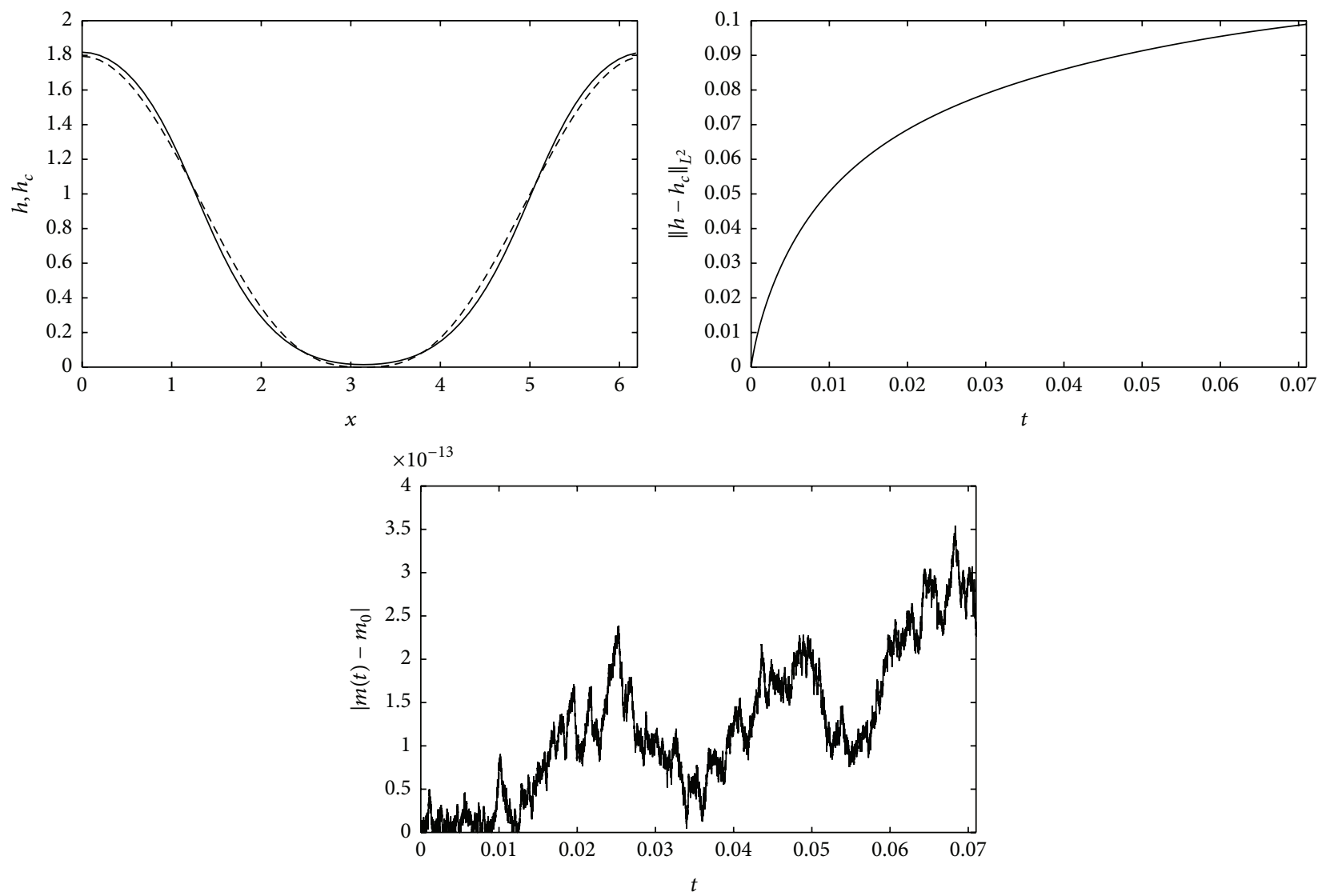

Figure 6: This time, the comparison of solutions to the equations is shown at the time when dashed line solution of (56) reaches touchdown point and at the same time the solid line solution of (4) retains its positivity for $n=1 / 2$ and $N=2^{6}$. Likewise, the $L^{2}$ norm of the difference of the solutions grows over time. Both numerical solutions are mass preserving.

periodic boundary conditions to implement a stable semiimplicit in time pseudospectral method described in [20].

\section{Discussion}

Modeling time evolution for different initial data, $h_{0}$, we noticed that the solutions of two different models, with nonlinear and linearized curvature terms, developed different shapes during the time evolution. When we applied our numerical simulations to the initial data for the large enough gradient value, namely, for

$$
h_{0}(x)=\frac{1}{0.09 \sqrt{2 \pi}} e^{-(x-\pi)^{2} / 2(0.09)^{2}},
$$

we observed that at the same time $t=10^{-4}$ the numerical solution for linearized model had lower height and more triangular shape to compare to the numerical solution for the nonlinear models which developed almost parallel sides of the node (see Figure 5 ), where $m(t)=\|h(\cdot, t)\|_{1}, m_{0}=m(0)$, and $h=h(x, t)$ for the linearized curvature model and $h_{c}=$ $h(x, t)$ for nonlinear curvature model.

It was also observed numerically that solutions of linearized and nonlinear curvature models approach zero (touchdown point) with a different speed; namely, the numerical solution for the linearized curvature model loses uniform positivity faster to compare to the numerical solution that corresponds to the nonlinear curvature model. To study the numerical time evolution of solutions near touchdown points, we implemented the numerical method suggested in [26] and used initial data $h_{0}(x)=0.8-\cos (\pi x)+0.25 \cos (2 \pi x)$ (see Figure 6).

\section{Competing Interests}

The authors declare that they have no competing interests.

\section{Acknowledgments}

This work was partially supported by a grant from the Simons Foundation (no. 275088 to Marina Chugunova).

\section{References}

[1] P. G. Saffman, "Viscous fingering in Hele-Shaw cells," Journal of Fluid Mechanics, vol. 173, pp. 73-94, 1986.

[2] B. E. Clothier, S. R. Green, and M. Deurer, "Preferential flow and transport in soil: progress and prognosis," European Journal of Soil Science, vol. 59, no. 1, pp. 2-13, 2008. 
[3] T. F. Dupont, R. E. Goldstein, L. P. Kadanoff, and S.-M. Zhou, "Finite-time singularity formation in Hele-Shaw systems," Physical Review E, vol. 47, article 4182, 1993.

[4] P. Constantin, T. F. Dupont, R. E. Goldstein, L. P. Kadanoff, M. J. Shelley, and S.-M. Zhou, "Droplet breakup in a model of the Hele-Shaw cell," Physical Review E, vol. 47, no. 6, pp. 4169-4181, 1993.

[5] R. Almgren, "Singularity formation in hele-shaw bubbles," Physics of Fluids, vol. 8, no. 2, article 344, 1996.

[6] A. Hernández-Machado, J. Soriano, A. M. Lacasta, M. A. Rodríguez, L. Ramírez-Plscina, and J. Ortín, "Interface roughening in Hele-Shaw flows with quenched disorder: experimental and theoretical results," Europhysics Letters, vol. 55, no. 2, pp. 194-200, 2001.

[7] C. Ruyer-Quil, "Inertial corrections to the Darcy law in a HeleShaw cell," Comptes Rendus de l'Academie des Sciences Series IIB Mechanics, vol. 329, no. 5, pp. 337-342, 2001.

[8] H.-G. Lee, J. S. Lowengrub, and J. Goodman, "Modeling pinchoff and reconnection in a Hele-Shaw cell. I. The models and their calibration," Physics of Fluids, vol. 14, no. 2, pp. 492$513,2002$.

[9] D. Crowdy, "Theory of exact solutions for the evolution of a fluid annulus in a rotating Hele-Shaw cell," Quarterly of Applied Mathematics, vol. 60, no. 1, pp. 11-36, 2002.

[10] M. Böckmann and S. C. Müller, "Growth rates of the buoyancydriven instability of an autocatalytic reaction front in a narrow cell," Physical Review Letters, vol. 85, no. 12, pp. 2506-2509, 2000.

[11] J. Martin, N. Rakotomalala, D. Salin, and M. Böckmann, "Buoyancy-driven instability of an autocatalytic reaction front in a Hele-Shaw cell," Physical Review E, vol. 65, no. 5, Article ID 051605, 2002.

[12] L. Giacomelli and F. Otto, "Rigorous lubrication approximation," Interfaces and Free Boundaries, vol. 5, no. 4, pp. 483-529, 2003.

[13] F. Bernis and A. Friedman, "Higher order nonlinear degenerate parabolic equations," Journal of Differential Equations, vol. 83, no. 1, pp. 179-206, 1990.

[14] E. A. Carlen and S. Ulusoy, "An entropy dissipation-entropy estimate for a thin film type equation," Communications in Mathematical Sciences, vol. 3, no. 2, pp. 171-178, 2005.

[15] E. A. Carlen and S. Ulusoy, "Asymptotic equipartition and long time behavior of solutions of a thin-film equation," Journal of Differential Equations, vol. 241, no. 2, pp. 279-292, 2007.

[16] F. Mohamed and A. Tudorascu, "Thin viscous films: thinning driven by surface-tension energy dissipation," Journal of Mathematical Analysis and Applications, vol. 431, no. 1, pp. 111-125, 2015.

[17] A. Tudorascu, "Lubrication approximation for thin viscous films: asymptotic behavior of nonnegative solutions," Communications in Partial Differential Equations, vol. 32, no. 7, pp. 11471172, 2007.

[18] M. Chugunova, J. R. King, and R. M. Taranets, "Uniqueness of the regular waiting-time type solution of the thin film equation," European Journal of Applied Mathematics, vol. 23, no. 4, pp. 537$554,2012$.

[19] M. Chugunova and R. M. Taranets, "New dissipated energy for nonnegative weak solution of unstable thin-film equations," Communications in Pure and Applied Analysis, vol. 10, no. 2, pp. 613-624, 2011.
[20] J. Becker, G. Grun, M. Lenz, and M. Rumpf, "Numerical methods for fourth order nonlinear degenerate diffusion problems," Applications of Mathematics, vol. 47, no. 6, pp. 517-543, 2002.

[21] M. Chugunova and R. Taranets, "Existence and long-time behaviour of weak solutions for the Mullins equation," Nonlinear Analysis: Theory, Methods \& Applications, vol. 75, no. 18, pp. 6550-6561, 2012.

[22] A. L. Bertozzi and M. Pugh, "The lubrication approximation for thin viscous films: the moving contact line with a 'porous media' cut-off of van der Waals interactions," Nonlinearity, vol. 7, no. 6, pp. 1535-1564, 1994.

[23] E. Beretta, M. Bertsch, and R. Dal Passo, "Nonnegative solutions of a fourth-order nonlinear degenerate parabolic equation," Archive for Rational Mechanics and Analysis, vol. 129, no. 2, pp. 175-200, 1995.

[24] E. DiBenedetto and A. Friedman, "Hölder estimates for nonlinear degenerate parabolic sytems," Journal für die Reine und Angewandte Mathematik, vol. 357, pp. 1-22, 1985.

[25] S. D. Eidelman, Parabolic Systems, North-Holland Publishing, Amsterdam, The Netherlands, 1969.

[26] A. L. Bertozzi, "The mathematics of moving contact lines in thin liquid films," Notices of the American Mathematical Society, vol. 45 , no. 6, pp. 689-697, 1998. 


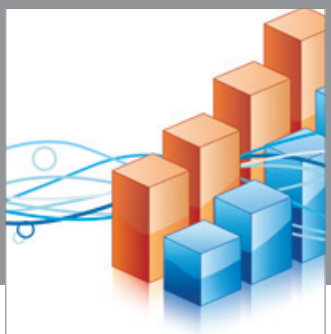

Advances in

Operations Research

vatem alat4

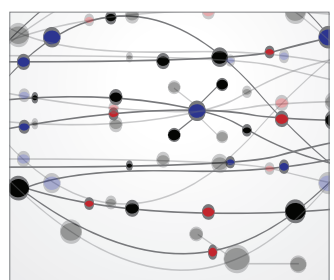

\section{The Scientific} World Journal
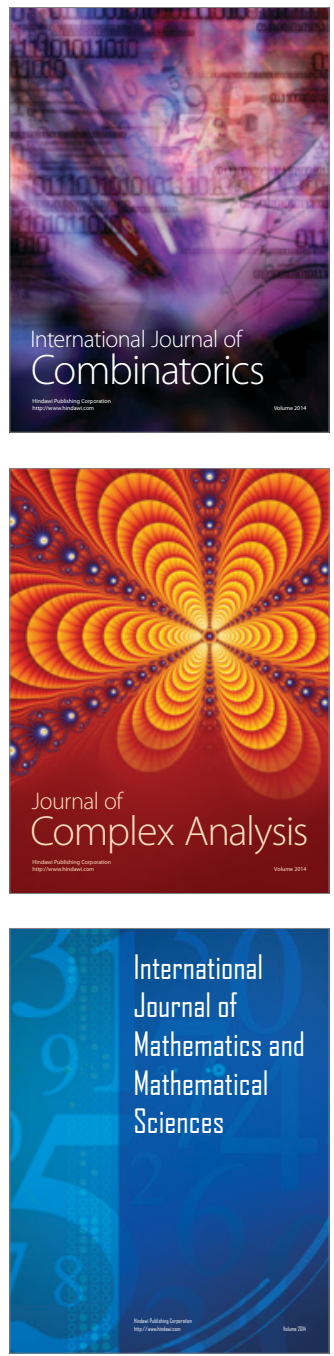
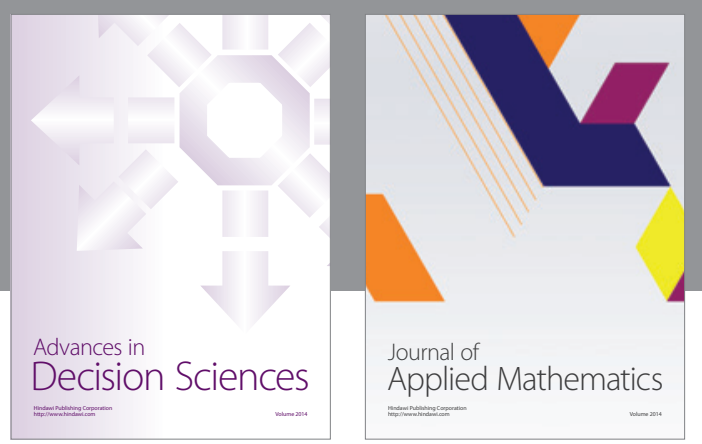

Algebra

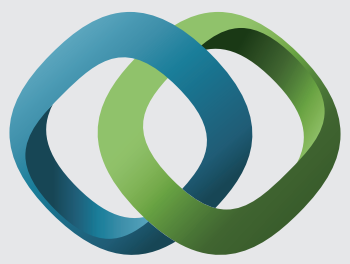

\section{Hindawi}

Submit your manuscripts at

http://www.hindawi.com
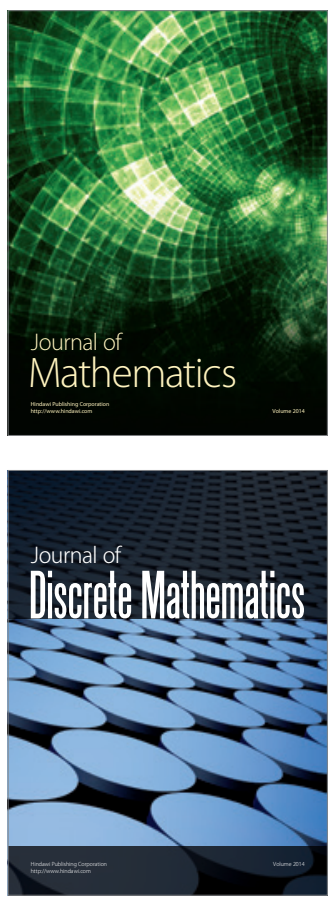

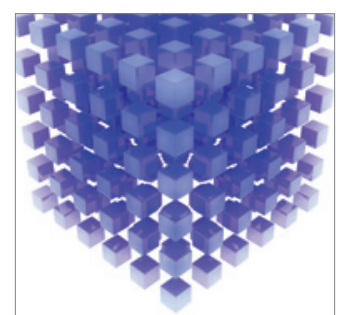

Mathematical Problems in Engineering
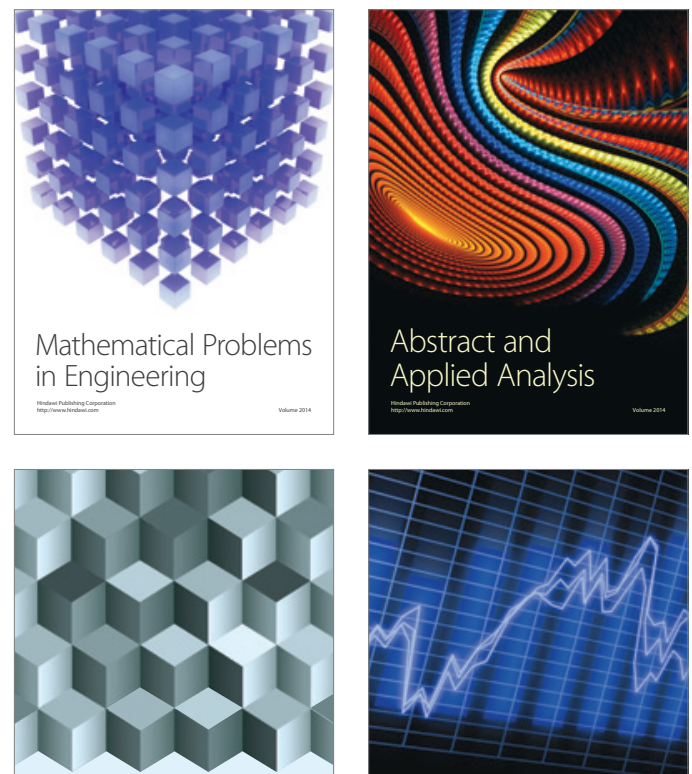

Journal of

Function Spaces

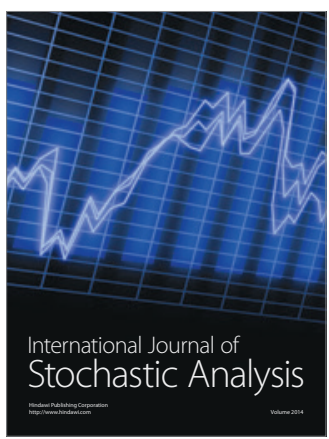

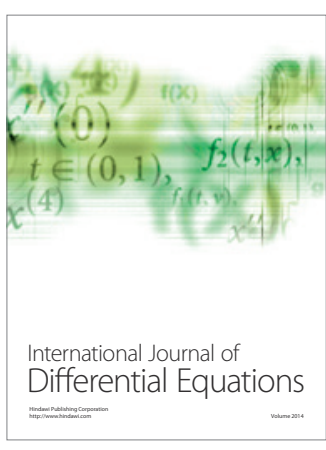
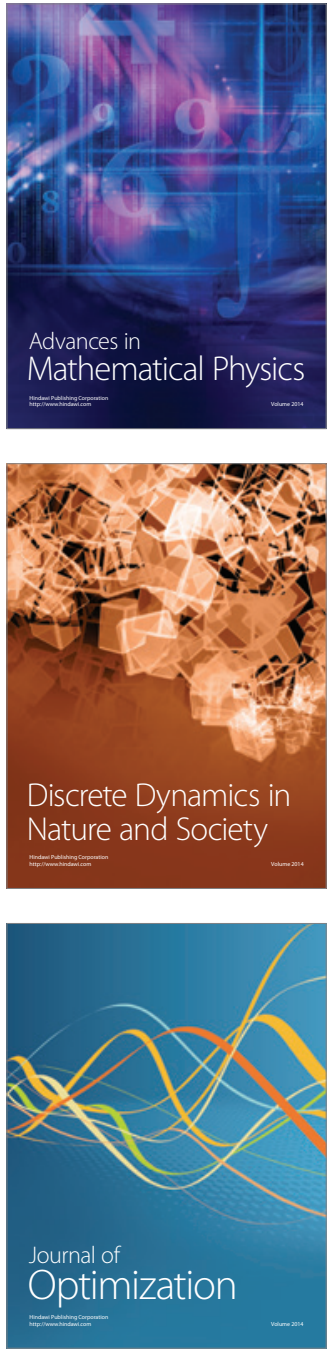